
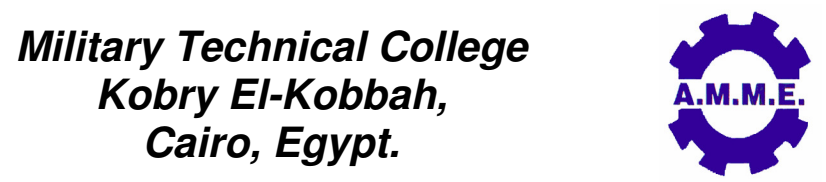
$15^{\text {th }}$ International Conference on Applied Mechanics and Mechanical Engineering.

\title{
NATURAL CONVECTION WITHIN AN ARBITRARILY ORIENTED ECCENTRIC ANNULUS
}

\author{
F. M. Mahfouz
}

\begin{abstract}
In this paper, the natural convection within an annulus formed between two eccentric circular cylinders has been numerically investigated. The annulus is placed horizontally and heated isothermally from its inner wall while its outer wall is isothermally cooled. The resulting laminar natural convection flow within the annulus is predicted at different orientations of the annulus symmetry line, ASL. The full governing equations are solved numerically using Fourier Spectral method. The process of heat convection within the annulus is dependent on Rayliegh number, Prandtl numbers, outer to inner cylinder radius ratio, eccentricity and angle of inclination of ASL to the horizontal, $\lambda$. The Prandtl number and radius ratio are fixed at 0.7 and 2.6, respectively. The Rayleigh number is taken within the range up to $10^{5}$, while $\lambda$ is varied from horizontal position $(\lambda=0)$ to vertical position $(\lambda=90)$. The results for mean Nussult number are obtained and discussed together with the details of both flow and thermal fields. The study has shown that for a given value of Rayleigh number as $\lambda$ increases the steady mean Nusselt number increases for negative eccentricities while it deceases for same but positive eccentricities. The study has also shown that for same RaL and $\lambda \neq 0$, the rate of heat transfer in the annulus is higher in case of negative eccentricities than that for same positive eccentricities.
\end{abstract}

\section{KEY WORDS}

Natural Convection, Constant Wall temperature, Fourier Spectral Method, Analytical solution.

\footnotetext{
* Associate professor, Dept. of Mech. Power, Menoufia University, Egypt.
} 


\section{NOMENCLATURE}

$c \quad h a l f$ distance between the two poles of Bi-polar coordinates

d $\quad \mathrm{c} / \mathrm{R}_{\mathrm{i}}$

E eccentricity

e dimensionless eccentricity $=\mathrm{E} /\left(R_{o}-R_{i}\right)$

$f_{n}, F_{n} \quad$ Fourier coefficients

$F_{b} \quad$ buoyancy force

g gravitational acceleration

$g_{n}, G_{n} \quad$ Fourier coefficients

$h, \bar{h} \quad$ local and average heat transfer coefficients

$h_{n}, H_{n} \quad$ Fourier coefficients

$J$ Jacobian of coordinate transformation

k thermal conductivity

Keq local Equivalent conductivity $(=N u \ln R r / 2)$

$\mathrm{L} \quad$ radius difference $=\left(R_{o}-R_{i}\right)$

$\mathrm{Nu}, \overline{\mathrm{Nu}} \quad$ local and mean Nusselt numbers

$\overline{N u}_{\text {cond }} \quad$ Conduction Nusselt number

$\operatorname{Pr} \quad$ Prandtl number $(=v / \alpha)$

$\mathrm{Rr} \quad$ radius ratio $=\left(R_{o} / R_{i}\right)$

RaL Rayleigh number based on $\mathrm{L}\left(=\mathrm{g} \beta(L)^{3}(\Delta T / \alpha v)\right.$

t dimensionless time

$T$ temperature

$\mathrm{x}^{\prime}, \mathrm{y}^{\prime} \quad$ Cartesian coordinates

$\mathrm{x}, \mathrm{y} \quad$ dimensionless Cartesian coordinates

\section{Greek symbols}

$\begin{array}{ll}\alpha & \text { Thermal diffusivity } \\ \beta & \text { Coefficient of thermal expansion } \\ \Delta T & \text { Reference temperature difference used in RaL }\left(=T_{i}-T_{o}\right) \\ \phi & \text { Dimensionless temperature }=T-T_{o} / \Delta T \\ \eta, \xi & \text { Bi-polar coordinates } \\ v & \text { Kinematics viscosity } \\ \rho & \text { Density } \\ \tau & \text { Time } \\ \psi^{\prime}, \psi & \text { dimensional and dimensionless stream functions } \\ \zeta^{\prime}, \zeta & \text { dimensional and dimensionless vorticity }\end{array}$




\section{Subscripts}

i at the inner wall
$0 \quad$ at the outer wall

\section{INTRODUCTION}

The problem of natural convection within an annular space between two concentric/eccentric cylinders has long been of both academic and practical interest because of its numerous engineering applications. These applications include solar collector-receivers, high-voltage electric transmission cables, cooling systems in nuclear reactors, and many others. Many experimental and numerical studies in case of natural convection between two concentric cylinders has been previously conducted. A good review of the relevant literatures has been given by Kuehn and Goldstein [1].

As compared with the concentric case, the volume of studies on natural convection between two eccentric cylinders is relatively small. Most of these studies, however, have mainly focused on the annulus between two vertically eccentric or horizontally eccentric cylinders. Kuehn and Goldstein [2], Chakrabarti et al.[3], badr [4], Prusa and Yao [5] to mention a few, studied the heat transfer in case of vertically eccentric annulus while Guj and Stella [6] and Guj et al. [7] studied the thermal field and heat transfer in horizontally eccentric annulus. In case of vertical eccentricity the annulus symmetry line, ASL (the line that connects the center of inner cylinder to the center of outer cylinder) is parallel to the gravity vector and hence the resulting flow field is symmetric with respect to the ASL and only half of the annulus can be considered as the computational domain. As a consequence, the stream function values can be set to zero on both the inner and outer cylinder walls. The numerical treatment of the problem in case of ASL being inclined with respect to the gravity vector is relatively cumbersome due to the possibility of having global flow circulation around the inner wall of the annulus. The existence of such a global flow within the annulus requires special numerical treatment of the boundary conditions of the stream function at the two walls of the annulus.

The natural global flow circulation within annular enclosures has been found to occur when the geometry of the enclosure is not symmetric with respect to gravity field. Such a global flow has been numerically predicted in case of inclined elliptic enclosure by Mahfouz [8]. In case of asymmetric eccentric annuli (if ASL is not vertical) a number of authors have studied the global flow within the annulus. Kuehn and Goldstein [2] experimentally predicted the global flow using the interferograms which were not clear enough to confirm whether the global circulation exists or not. Wang and Bau [9] also studied the flow field using a perturbation expansion. On the other side, Cho et al. [10] and Projahn et al. [11] investigated numerically the problem using vorticity-stream function formulation, but they assigned zero values to the stream function on the two walls of the annulus which prevents the numerical prediction of the global flow in the annulus. This treatment is a good approximation only in the case of small eccentricity but can not be used for large eccentricities 
where the stream function values on the two walls are quite different because of the global flow circulation $[12,13]$.

Natural convection in case of asymmetric annuli has been further considered by a number of authors who applied different numerical techniques to investigate the validity of such techniques to solve the problem. Shu et al. [14] used polynomialbased differential quadrature (PDQ) and Fourier expansion-based differential quadrature (FDQ) while Shu and Wu [15] used the domain-free discretization (DFD) method. Lee et. al. [16] used a generalized differential-integral quadrature (GDQ) technique to discretize the natural convection governing equations using a bodyfitted coordinate system.

It can be observed that most of the previous studies focused on the steady natural convection within vertically eccentric annuli, Moreover, most of the studies that considered asymmetric annuli have focused on plotting the flow and thermal fields without paying enough attention to the effect of inclination of ASL on heat transfer at different eccentricities. So the objective of this study is to develop an accurate numerical technique, using bi-polar orthogonal coordinate transformation together with the spectral Fourier method, to further extend the existing knowledge on natural convection heat transfer in arbitrarily oriented eccentric annuli.

\section{PROBLEM FORMULATION}

The physical domain under consideration, shown in Fig.1, is the annulus formed between two long horizontal eccentric cylinders. The outer cylinder has radius $R_{o}$, while the inner cylinder has radius $R_{i}$, and is placed in such away the line passing through its center and through the center of outer cylinder inclines with angle $\lambda$ to the horizontal. The eccentricity, $\mathrm{E}$ between the two cylinders is taken positive if the center of the inner cylinder is above that of the outer cylinder (or to the right of it ) and vice versa. The annulus contains incompressible, Newtonian fluid and is heated through its inner wall which is maintained at constant wall temperature, $T_{i}$ while the outer wall is cooled and kept at constant temperature $T_{o}$ higher than that of the cooling ambient. The resulting buoyancy driven flow in the annulus is assumed to be laminar and two dimensional. The density of the fluid is considered constant except in the buoyancy term where it varies according to Boussinesq approximation. Moreover, viscous dissipation, internal heat generation and radiation heat transfer are either absent or neglected.

\section{The Governing Equations}

Immediately, after heating the annulus through its inner wall buoyancy driven flow is initiated. The induced flow has been initially time dependent and the steady state condition is reached after a period of time. The time dependent conservation equations of mass, momentum and energy can be written in Cartesian coordinates in terms of stream function, vorticity and temperature as: 


$$
\begin{aligned}
& \frac{\partial \zeta^{\prime}}{\partial \tau}=v\left(\frac{\partial^{2} \zeta^{\prime}}{\partial x^{\prime 2}}+\frac{\partial^{2} \zeta^{\prime}}{\partial y^{\prime 2}}\right)-\frac{\partial \psi^{\prime}}{\partial y^{\prime}} \frac{\partial \zeta^{\prime}}{\partial x^{\prime}}+\frac{\partial \psi^{\prime}}{\partial x^{\prime}} \frac{\partial \zeta^{\prime}}{\partial y^{\prime}}-\frac{1}{\rho}\left(\frac{\partial F_{x^{\prime}}}{\partial y^{\prime}}-\frac{\partial F_{y^{\prime}}}{\partial x^{\prime}}\right) \\
& \zeta^{\prime}=-\left(\frac{\partial^{2} \psi^{\prime}}{\partial x^{\prime 2}}+\frac{\partial^{2} \psi^{\prime}}{\partial y^{\prime 2}}\right) \\
& \frac{\partial T}{\partial \tau}=\alpha\left(\frac{\partial^{2} T}{\partial x^{\prime 2}}+\frac{\partial^{2} T}{\partial y^{\prime 2}}\right)-\frac{\partial \psi^{\prime}}{\partial y^{\prime}} \frac{\partial T}{\partial x^{\prime}}+\frac{\partial \psi^{\prime}}{\partial x^{\prime}} \frac{\partial T}{\partial y^{\prime}}
\end{aligned}
$$

where $\tau$ is the time, $\psi^{\prime}$ is the stream function, $\zeta^{\prime}$ is the vorticity, $T$ is the temperature, $\rho$ is the density, $v$ and $\alpha$ are the momentum and thermal diffusivities respectively. $F_{x^{\prime}}=\rho g \beta\left(T-T_{o}\right) \sin (\lambda), F_{y^{\prime}}=\rho g \beta\left(T-T_{o}\right) \cos (\lambda)$ are the components of the net upward driving force, $F_{b}$.

\section{Initial and Boundary Conditions}

At time $\mathrm{t}=\mathrm{0}^{+}$, the inner wall is assumed to be impulsively heated to constant wall temperature while the outer wall is kept at fixed temperature which is the same as the initial temperature of initially stagnant fluid. The hydrodynamics boundary conditions at the inner and outer walls are the no-slip condition and impermeability. The thermal boundary conditions at the inner and outer walls are the constant wall temperature, $T_{i}, T_{o}$ respectively, with $T_{i}>T_{o}$. The steady state boundary conditions can be expressed mathematically as follows:

-On the outer wall

$$
\psi^{\prime}=\frac{\partial \psi^{\prime}}{\partial x^{\prime}}=0, \quad \frac{\partial \psi^{\prime}}{\partial y^{\prime}}=0 \text { and } T=T_{o}
$$

- On the inner wall

$$
\frac{\partial \psi^{\prime}}{\partial x^{\prime}}=0, \frac{\partial \psi^{\prime}}{\partial y^{\prime}}=0, \text { and } \quad T=T_{i}
$$

The stream function, as indicated above, is set to zero on the outer wall while its value on the inner wall will be calculated. The calculated value of the stream function at the inner wall will determine both intensity and direction of the global flow circulation around the inner wall, if exists.

\section{Coordinate Transformation}

In order to set the inner and outer walls boundary conditions properly without interpolation, a coordinate system that makes the boundary lines coincide with coordinate's lines should be used. In this problem the bipolar coordinates $\xi, \eta$ defined as $x=-d \sinh \xi /(\cosh \xi-\cos \eta), \quad y=d \sin \eta /(\cosh \xi-\cos \eta)$ is adopted. In bipolar coordinates the governing equations (1-3) read: 


$$
\begin{gathered}
J \frac{\partial \zeta}{\partial t}=\operatorname{Pr}\left(\frac{\partial^{2} \zeta}{\partial \xi^{2}}+\frac{\partial^{2} \zeta}{\partial \eta^{2}}\right)+\left(\frac{\partial \psi}{\partial \xi} \frac{\partial \zeta}{\partial \eta}-\frac{\partial \psi}{\partial \eta} \frac{\partial \zeta}{\partial \xi}\right) \\
+\frac{\operatorname{Pr} R a L}{d^{*}(R r-1)^{3}} \sin (\lambda)\left[J(\cosh \xi \cos \eta-1) \frac{\partial \phi}{\partial \eta}-J\left(\sinh \xi \sin \eta \frac{\partial \phi}{\partial \xi}\right]\right. \\
-\frac{\operatorname{Pr} R a L}{d^{*}(R r-1)^{3}} \cos (\lambda)\left[J(\cosh \xi \cos \eta-1) \frac{\partial \phi}{\partial \xi}+J\left(\sinh \xi \sin \eta \frac{\partial \phi}{\partial \eta}\right]\right. \\
J \zeta=\frac{\partial^{2} \psi}{\partial \xi^{2}}+\frac{\partial^{2} \psi}{\partial \eta^{2}} \\
J \frac{\partial \phi}{\partial t}=\left(\frac{\partial^{2} \phi}{\partial \xi^{2}}+\frac{\partial^{2} \phi}{\partial^{2} \eta}\right)+\frac{\partial \psi}{\partial \xi} \frac{\partial \phi}{\partial \eta}-\frac{\partial \psi}{\partial \eta} \frac{\partial \phi}{\partial \xi}
\end{gathered}
$$

where

$$
x=x^{\prime} / R_{i}, y=y^{\prime} / R_{i}, \quad d=c / R_{i}, \quad t=\tau \alpha / R_{i}^{2}, \quad \psi=\psi^{\prime} / \alpha, \quad \zeta=-\zeta^{\prime} R_{i}^{2} / \alpha \quad \text { and }
$$

$\phi=\left(T-T_{o}\right) / \Delta T . J=x_{\xi} y_{\eta}-x_{\eta} y_{\xi}$ is the determinant of the Jacobian of coordinate transformation matrix, $\operatorname{Pr}=v / \alpha$ is the Prandtl number, $R a L=g \beta R_{i}^{3}(R r-1)^{3} \Delta T / v \alpha$ is the Rayleigh number and $\Delta T$ is the temperature difference $=T_{i}-T_{o}$.

In the new coordinates, the steady boundary conditions (4) can now be expressed as:

- On the outer cylinder surface, $\xi=\xi_{o}$

$$
\psi=\frac{\partial \psi}{\partial \xi}=0, \frac{\partial \psi}{\partial \eta}=0 \quad \text { and } \quad \phi=0
$$

- On the inner cylinder surface, $\xi=\xi_{i}$

$$
\frac{\partial \psi}{\partial \xi}=0, \frac{\partial \psi}{\partial \eta}=0 \quad \text { and } \quad \phi=1
$$

where $\xi_{i}$ and $\xi_{o}$ are negative constants that define the inner and outer walls surfaces $\left(\xi_{i}=\sinh ^{-1}(-d), \xi_{o}=\sinh ^{-1}(-d / R r)\right)$ where $R r=R_{o} / R_{i}$ is the radius ratio.

\section{METHOD OF SOLUTION AND NUMERICAL PROCEDURE}

The method of solution is based on Fourier spectral method in which the dependent variables stream function $\psi$, vorticity $\zeta$ and temperature $\phi$ are approximated using Fourier series expansion. The approach is similar to that used by Badr and Dennis [17] and Mahfouz and Badr [18]. The dimensionless stream function, vorticity and temperature are approximated as: 


$$
\begin{aligned}
& \psi(\xi, \eta, t)=\frac{1}{2} \mathrm{~F}_{\mathrm{o}}(\xi, t)+\sum_{n=1}^{N}\left[f_{n}(\xi, t) \sin (n \eta)+F_{n}(\xi, t) \cos (n \eta)\right] \\
& \zeta(\xi, \eta, t)=\frac{1}{2} \mathrm{G}_{\mathrm{o}}(\xi, t)+\sum_{n=1}^{N}\left[g_{n}(\xi, t) \sin (n \eta)+G_{n}(\xi, t) \cos (n \eta)\right] \\
& \phi(\xi, \eta, t)=\frac{1}{2} \mathrm{H}_{\mathrm{o}}(\xi, t)+\sum_{n=1}^{N}\left[h_{n}(\xi, t) \sin (n \eta)+H_{n}(\xi, t) \cos (n \eta)\right]
\end{aligned}
$$

where $F_{0}, f_{n}, F_{n}, G_{0}, g_{n}, G_{n}, H_{0}, h_{n}$, and $H_{n}$ are the Fourier coefficients and $N$ represents the number of terms considered in the Fourier series. Substitution of $\psi$, $\zeta$ and $\phi$ defined in (9) in equations (5-7) and integrating these equations (after multiplying each by $1, \sin (n \eta)$ and $\cos (n \eta)$ respectively) from 0 to $2 \pi$ results in the following set of differential equations:

$$
\begin{aligned}
& \frac{\partial^{2} F_{o}}{\partial \xi^{2}}=a_{o} G_{o}+\sum_{1}^{N} a_{m} G_{m} \\
& \frac{\partial^{2} F_{n}}{\partial \xi^{2}}-n^{2} F_{n}=\frac{1}{2} a_{n} G_{o}+a_{o} G_{n}+\frac{1}{2} \sum_{1}^{N} G_{m}\left(a_{n+m}+a_{|m-n|}\right) \\
& \frac{\partial^{2} f_{n}}{\partial \xi^{2}}-n^{2} f_{n}=a_{o} g_{n}+\frac{1}{2} \sum_{1}^{N} g_{m}\left(a_{|m-n|}-a_{n+m}\right) \\
& a_{o} \frac{\partial G_{o}}{\partial t}+\sum_{1}^{N} a_{m} \frac{\partial G_{m}}{\partial t}=\operatorname{Pr} \frac{\partial^{2} G_{o}}{\partial \xi^{2}}+S G_{o}(\xi, t) \\
& a_{o} \frac{\partial G_{n}}{\partial t}+\frac{1}{2} \frac{\partial G_{o}}{\partial t} a_{n}+\frac{1}{2} \sum_{1}^{N} \frac{\partial G_{m}}{\partial t}\left(a_{n+m}+a_{|n-m|}\right)=\operatorname{Pr}\left(\frac{\partial^{2} G_{n}}{\partial \xi^{2}}-n^{2} G_{n}\right)+S G_{n}(\xi, t) \\
& a_{o} \frac{\partial g_{n}}{\partial t}+\frac{1}{2} \sum_{1}^{N} \frac{\partial g_{m}}{\partial t}\left(a_{|m-n|}-a_{n+m}\right)=\operatorname{Pr}\left(\frac{\partial^{2} g_{n}}{\partial \xi^{2}}-n^{2} g_{n}\right)+S g_{n}(\xi, t) \\
& a_{o} \frac{\partial H_{o}}{\partial t}+\sum_{1}^{N} a_{m} \frac{\partial H_{m}}{\partial t}=\frac{\partial^{2} H_{o}}{\partial \xi^{2}}+Z H_{o}(\xi, t) \\
& a_{o} \frac{\partial H_{n}}{\partial t}+\frac{1}{2} \frac{\partial H_{o}}{\partial t} a_{n}+\frac{1}{2} \sum_{1}^{N} \frac{\partial H_{m}}{\partial t}\left(a_{n+m}+a_{|n-m|}\right)=\left(\frac{\partial^{2} H_{n}}{\partial \xi^{2}}-n^{2} H_{n}\right)+Z H_{n}(\xi, t) \\
& a_{o} \frac{\partial h_{n}}{\partial t}+\frac{1}{2} \sum_{1}^{N} \frac{\partial h_{m}}{\partial t}\left(a_{|m-n|}-a_{n+m}\right)=\left(\frac{\partial^{2} h_{n}}{\left.\partial \xi^{2}-n^{2} h_{n}\right)+Z h_{n}(\xi, t)}\right.
\end{aligned}
$$

where $a_{o}, a_{n}$ are the coefficients that result from expanding the term $\mathrm{J}$ in the form $\left(a_{o}+\sum_{n=1}^{\infty} a_{n} \cos n \eta\right) . \mathrm{SG}_{0}, \mathrm{SG}_{\mathrm{n}}, \mathrm{Sg}_{\mathrm{n}}, \mathrm{ZH}_{\mathrm{o}}, \mathrm{ZH}_{\mathrm{n}}$ and $\mathrm{Zh}_{\mathrm{n}}$ are all easily identifiable functions. Equations (10)-(12) define a system of differential equations that should be solved simultaneously at every time step. 
The steady boundary conditions for all Fourier functions presented in equations (1012) are deduced from (8) and can be expressed as:

$$
\begin{aligned}
& \text { - At } \xi=\xi_{0} \\
& \mathrm{f}_{\mathrm{n}}=\partial \mathrm{f}_{\mathrm{n}} / \partial \xi=\mathrm{F}_{0}=\partial \mathrm{F}_{0} / \partial \xi=\mathrm{F}_{\mathrm{n}}=\partial \mathrm{F}_{\mathrm{n}} / \partial \xi=0, \mathrm{H}_{\mathrm{n}}=\mathrm{h}_{\mathrm{n}}=0 \text { and } \mathrm{H}_{0}=0 \\
& \text { - At } \xi=\xi_{\mathrm{i}} \\
& \mathrm{f}_{\mathrm{n}}=\partial \mathrm{f}_{\mathrm{n}} / \partial \xi=\partial \mathrm{F}_{\mathrm{o}} / \partial \xi=\mathrm{F}_{\mathrm{n}}=\partial \mathrm{F}_{\mathrm{n}} / \partial \xi=0, \mathrm{H}_{\mathrm{n}}=\mathrm{h}_{\mathrm{n}}=0 \text { and } H_{o}=2
\end{aligned}
$$

From the condition at inner wall (13b) it can be concluded that the value of stream function at the inner wall will be equal to $\psi_{\text {wall }}=F_{o(\text { wall })} / 2$. The value of $\mathrm{F}_{\mathrm{o}}$ at the wall is determined from the solution of eq.(10a). The vorticity distribution at inner wall is found from the following integral conditions that developed from the integration of eq. (10) from $\xi=\xi_{\mathrm{i}}$ to $\xi=\xi_{0}$ and using boundary conditions (13).

$$
\begin{aligned}
& \int_{\xi_{i}}^{\xi_{o}}\left(a_{o} G_{o}+\sum_{1}^{N} a_{m} G_{m}\right) d \xi=0 \\
& \int_{\xi_{i}}^{\xi_{o}}\left(\frac{1}{2} a_{n} G_{o}+a_{o} G_{n}+\frac{1}{2} \sum_{1}^{N} G_{m}\left(a_{n+m}+a_{|m-n|}\right)\right) e^{-n \xi} d \xi=0 \\
& \int_{\xi_{i}}^{\xi_{o}}\left(a_{o} g_{n}+\frac{1}{2} \sum_{1}^{N} g_{m}\left(a_{|m-n|}-a_{n+m}\right)\right) e^{-n \xi} d \xi=0
\end{aligned}
$$

The above integrals are used to calculate the values of the functions $G_{0}, G_{n}, g_{n}$ on the inner wall surface from which the vorticity distribution along the wall is predicted. The vorticity distribution at outer wall is calculated at $\left(\xi=\xi_{0}\right)$ from eq. (10).

The numerical analysis proceeds further by discretizing the ordinary differential equation (10) and partial differential equations (11-12) using finite difference method. In discretization process all spatial derivatives are approximated using second order accurate central finite difference scheme. The first derivatives at the inner and outer walls are approximated by one side second order accurate forward (at inner wall) or backward (at outer wall) three point finite difference scheme. The discretization of time dependent equations (11-12) is handled using Crank-Nicolson scheme. The forward time step is taken very small to achieve the accuracy of the scheme. At every time step the coupled non-linear system of equations has been linearized through an iterative procedure, resulting in tri-diagonal system of equations that has been solved by Tri-Diagonal Matrix Algorithm, TDMA. More details about solution procedure are given by Badr and Dennis [17] and Mahfouz and Badr [18].

\section{Heat Transfer Parameters}

After obtaining the distributions of stream function and temperature, the flow and 
heat transfer characteristics can be easily determined. The stream function distribution facilitates plotting the flow field in terms of streamlines and also enables determining the flow velocity. While the temperature distribution helps find the heat transfer results in terms of local and mean Nusselt numbers.

The local and mean Nussult numbers are the direct indicator of local heat flux and mean rate of heat transfer at the walls. The local Nusselt numbers at the inner and outer walls are defined as $N u_{i}=2 h_{i} R_{i} / k$ and $N u_{o}=2 h_{o} R_{o} / k$, where $\mathrm{k}$ is the fluid thermal conductivity, and $h_{i}$ and $h_{0}$ are the local heat transfer coefficients at inner and outer walls and are defined as:

$$
\begin{array}{ll}
h_{i}=q_{i} / \Delta T_{r e f} ; & h_{o}=q_{o} / \Delta T_{r e f} \\
q_{i}=-k\left(\partial T / \partial S_{n}\right)_{i} & q_{o}=-k\left(\partial T / \partial S_{n}\right)_{o}
\end{array}
$$

$S_{n}$ is the local direction of heat flux normal to the wall. From the above definitions one can deduce

$$
N u_{i}=-2\left(J^{-1 / 2} \frac{\partial \phi}{\partial \xi}\right)_{i} ; \quad N u_{o}=-2 \operatorname{Rr}\left(J^{-1 / 2} \frac{\partial \phi}{\partial \xi}\right)_{o}
$$

The mean Nusselt number is defined as $\overline{N u}=\frac{1}{p} \int_{0}^{P} N u d p$ where $\mathrm{P}$ is the perimeter of the circular section. The mean Nusselt number at inner and outer walls can now be expressed as:

$$
\overline{N u}_{i}=-\left(\frac{\partial H_{o}}{\partial \xi}\right)_{i} ; \quad \overline{N u}_{o}=-\left(\frac{\partial H_{o}}{\partial \xi}\right)_{o}
$$

Based on the above definition of mean Nusselt number at inner and outer walls it can be easily concluded that the ratio of mean Nusselt number at the inner and outer walls is equal to the ratio of the total rate of heat transfer from the two walls, that is $\overline{N u}_{i} / \overline{N u}_{o}=\dot{Q}_{i} / \dot{Q}_{o}$. Based on energy conservation within the annulus, it can be easily concluded that in the steady state condition the heat transfer rate into the annulus through the inner wall must be equal to the heat transfer rate rejected out the annulus through the outer wall. Thus when the mean Nussult number at outer wall approaches that at inner wall (within tolerance of 0.001 ) the steady state solution is reached and the calculation is terminated.

For steady state pure conduction it is proved, through the analytical solution of energy equation, that the mean Nusselt number can be expressed as :

$$
\overline{N u}_{\text {cond }}=2 / \cosh ^{-1}\left(\frac{R_{o}{ }^{2}+R_{i}{ }^{2}-E^{2}}{2 R_{o} R_{i}}\right)
$$




\section{RESULTS AND DISCUSSION}

Before producing the numerical results the validity of mathematical model and numerical technique has been first assessed by conducting independency tests for both grid size in $\xi$ direction and the number of terms considered in the Fourier series as well as performing many comparisons with the previous numerical and experimental relevant studies. Some of these comparisons are presented wherever adequate throughout the paper. These tests and comparisons have shown that the present numerical solution is highly accurate. Once the numerical solution has been validated, a set of runs is carried out to study the effect of controlling parameters on both flow and thermal fields within the annulus. The main controlling parameters beside the classical ones ( $\mathrm{RaL}$ and $\mathrm{Pr}$ ) are the angle of inclination of ASL with respect to horizontal, $\lambda$ the eccentricity, $e$ and the radius ratio, $\mathrm{Rr}$. The study considered a moderate range of RaL up to $10^{5}$ while Prandtl number is fixed at 0.7 . The angle $\lambda$ is varied from $0^{\circ}$ to $90^{\circ}$ and the eccentricity is varied between -0.65 and +0.65 while the radius ratio is fixed at 2.6.

The numerical solution, as mentioned, has marched in time till reaching the steady state solution. The steady state solution is reached when the total heat rejected from the outer wall of the annulus approaches that pumped into the annulus through the inner wall. In this case the steady state solution is obtained when $\overline{N u}_{o}$ is equal to $\overline{N u}_{i}$ as can be seen in Fig. 2 which shows the time development of $\overline{N u}_{i}$ and $\overline{N u}_{o}$ along inner and outer walls when ASL is vertical $(\lambda=90)$ and when ASL is horizontal $(\lambda=0)$ at $\mathrm{RaL}=5 \times 10^{3}, \mathrm{Rr}=2.6$ and $\mathrm{e}=+0.5$. The figure shows that the rate of heat rejected (in terms of $\overline{N u}_{o}$ ) from outer wall increases with time while that pumped (in terms of $\overline{N u}_{i}$ ) into the annulus through the inner wall decreases until they become equal at the steady state. It can also be seen that in the very initial time stages the time development of $\overline{N u}_{i}$ is identical for the two cases of $\lambda=90$ and $\lambda=0$. This is because in initial time stages the heat transmission occurs only by conduction through very thin layer adjacent to inner wall and no role for convection. Also it can be seen that the time period taken to reach to the steady state condition as well as the steady state value of mean Nu are dependent on ASL inclination angle $\lambda$.

The patterns of steady state streamlines and isotherms in case of symmetrical annulus $\lambda=90$ are shown in Fig. 3 while those in case of an asymmetric annulus $\lambda=$ 0 are shown in Fig.4. It can be seen in symmetric case that both the flow field, Fig.3a, and the thermal field, Fig. 3b, are symmetric with respect to ASL. Fig. 3a shows that the flow field consists of two similar eddies one on each side of the annulus. These two eddies are separated by zero streamline line (dashed line) which coincides in this case with inner and outer walls and ASL line as well. In case of asymmetric field, Fig.4b, the two eddies are no longer being similar but rather have different sizes. In this case the zero streamline coincide with neither the inner wall nor the ASL but rather shifted away from the inner wall, indicating the existence of global flow circulation around the inner wall. The global flow was found to join the eddy in the larger side of the annulus. In this case, the imbalance between induced flow in the two sides of the annulus creates a global flow that circulates around the 
inner wall as shown in the figure. Moreover, the thermal field in case of asymmetric case, Fig. 4a shows the formation and inclination of thermal plume in the direction of flow that comes upward and leaves the hot inner wall towards the cold outer wall. Moreover, the asymmetry of thermal field is further inspected in Fig.5 which shows the heat flux distribution (in terms of local $\mathrm{Nu}$ ) at the inner and outer walls of the annulus in case of $\lambda=90$ ( Fig.5a) and in case of $\lambda=0$ (Fig. 5b). Unlike the symmetrical case $(\lambda=90)$, the figure shows that the distribution of heat flux in case of $\lambda=0$ is not symmetrical with respect to ASL.

Further inspection of Fig. 5a shows that the minimum heat flux (Nu) along the outer wall occurs in case of $\lambda=90$ at the point of flow separation from the wall at $\theta=0$ while the maximum $\mathrm{Nu}$ occurs at $\theta \approx 125$. For the inner wall, the maximum $\mathrm{Nu}$ occurs at the point of flow stagnation at the lowest point of the wall at $\theta=0$ while the minimum Nu occurs at $\theta \approx 125$. In case of $\lambda=0$ the inspection of Fig. $5 \mathrm{~b}$ shows that the maximum Nu along the outer wall occurs at the point of flow stagnation with the wall at $\theta \approx 80$ while the maximum Nu along the inner wall occurs at $\theta \approx 335$.

Table 1 shows the effect of controlling parameters on $\overline{\mathrm{Nu}}$. Shown also in the table are the numerical and analytical value for steady mean Nusselt number pure conduction $(R a L=0)$. It can be seen that in case of $R a L=0$ both numerical and analytical values are almost the same which assesses the accuracy of the numerical solution. In case of considering convection effects $(\mathrm{RaL} \neq 0)$ the effect of $\mathrm{RaL}$ on $\overline{\mathrm{Nu}}$ is clear and as expected, that is as RaL increases the $\overline{N u}$ increases for all inclinations and eccentricities as a result of increasing the convection currents intensity which enhances heat transfer coefficient and so increases the heat transfer rate.

The effect of eccentricity e on $\overline{N u}$ can also be observed in Table 1 . In case of RaL $=0$ the table shows that the heat transfer rate (in terms of $\overline{\mathrm{Nu}}$ ) is minimum in case of $\mathrm{e} \approx 0$ (i.e almost concentric annulus) and increases as the absolute value of $e$ increases ( from 0 to 1). In case of considering convection effects the table shows that the heat transfer rate in case of any negative eccentricity is bigger than that for same but positive eccentricity. This can be roughly explained by considering the heat convection between two parallel horizontal plates with one plate heated and the other plate cooled. It is well known that if the heated plate is facing up (and cooled plate is facing down) the convection process becomes more effective than that if the heated plate is facing down (and cooled plate is facing up). In this latter case the temperature gradient is not adequate for active convection and the heat transfer is mainly due to conduction. In case of eccentric cylinders the most upper part of the heated inner cylinder is facing up and the most upper part of the cold outer cylinder is facing down while the most lower part of the heated inner wall is facing down and the most lower part of the cold outer wall is facing up. In such configuration the heat convection from the upper part of inner wall will be more as compared with that from the lower part. Therefore, the total heat transfer from the inner wall is controlled by the effectiveness of heat convection in the gap between the most upper part of the inner wall and the outer wall. The wider this gap the more efficient the heat 
Table 1. Effect of RaL, $\lambda$ and e on steady $\overline{N u}$.

\begin{tabular}{|c|c|c|c|c|c|c|}
\hline \multirow{3}{*}{ RaL } & \multirow{6}{|c|}{$N u$} \\
\cline { 3 - 7 } & $\lambda$ & $\mathrm{e}=-0.60$ & $\mathrm{e}=-0.25$ & $\mathrm{e} \approx 0.0$ & $\mathrm{e}=0.25$ & $\mathrm{e}=0.60$ \\
\hline \hline \multirow{3}{*}{0.0} & -- & $\begin{array}{c}2.583 \\
(2.583)^{\star}\end{array}$ & $\begin{array}{c}2.157 \\
(2.157)^{\star}\end{array}$ & $\begin{array}{c}2.092 \\
(2.093)^{\star}\end{array}$ & $\begin{array}{c}2.157 \\
(2.157)^{\star}\end{array}$ & $\begin{array}{c}2.583 \\
(2.583)^{*}\end{array}$ \\
\hline \multirow{4}{*}{$10^{3}$} & 0 & 2.901 & 2.382 & 2.267 & 2.382 & 2.901 \\
\cline { 3 - 7 } & 30 & 2.989 & 2.412 & 2.267 & 2.349 & 2.842 \\
\cline { 2 - 7 } & 60 & 3.062 & 2.445 & 2.267 & 2.329 & 2.799 \\
\cline { 2 - 7 } & 90 & 3.095 & 2.459 & 2.267 & 2.319 & 2.775 \\
\hline \multirow{4}{*}{$10^{4}$} & 0 & 4.125 & 4.100 & 4.156 & 4.100 & 4.125 \\
\cline { 2 - 7 } & 30 & 4.335 & 4.196 & 4.156 & 4.016 & 3.911 \\
\cline { 2 - 7 } & 60 & 4.515 & 4.271 & 4.156 & 3.931 & 3.858 \\
\cline { 2 - 7 } & 90 & 4.572 & 4.301 & 4.156 & 3.899 & 3.825 \\
\hline \multirow{4}{*}{$10^{5}$} & 0 & 7.000 & 7.219 & 7.261 & 7.219 & 7.000 \\
\cline { 2 - 7 } & 30 & 7.110 & 7.284 & 7.261 & 7.125 & 6.721 \\
\cline { 2 - 7 } & 60 & 7.292 & 7.386 & 7.261 & 7.085 & 6.481 \\
\cline { 2 - 7 } & 90 & 7.542 & 7.432 & 7.261 & 7.025 & 6.348 \\
\hline \hline
\end{tabular}

* Analytical $\overline{\mathrm{Nu}}_{\text {cond }}$

convection process. In case of negative eccentricity the wide upper gap between the two walls helps enhance heat convection as a result of intensive motion of convection currents. While for same but positive eccentricity the narrow upper gap between the two walls hinders the convection currents and results in small convection contribution.

Table 1 further shows the effect of ASL inclination angle $\lambda$ on $\overline{N u}$. It can be seen that as $\lambda$ increases $\overline{N u}$ increases in case of negative eccentricities while it deceases in case of positive eccentricities. In case of negative eccentricity as the angle of ASL increases from zero to 90 the upper gap between the two walls gets wider and so enhances heat convection process. While for same but positive eccentricity as the angle of ASL increases the upper gap between the two walls gets narrower which reduces heat convection.

For the purpose of comparison with previous research, the flow field in terms of streamlines and thermal field in terms of isotherms are shown for the symmetrical case of vertically eccentric annulus $(\mathrm{e}=+0.652$ ) in Fig. 6 . The figure clearly shows that in this case of positive eccentricity the flow and thermal fields are characterized by weak convection currents in the upper part and formation of a nearly isothermal stagnant region in the lower part of the annulus. Shown also in the same figure are the full pattern of experimental isotherms of Kuehn and Goldstein [2] and the numerical results of Chung et al [19] which are presented as one half of the isotherms pattern and one half of the corresponding streamlines. The figure shows 
good qualitative agreement between the present results and those presented in aforementioned refs. [2, 19].

The present equivalent conductivity distributions along the inner and outer walls for the abovementioned case are shown in Fig. 7. The equivalent conductivity, Keq is defined as the actual heat flux divided by the heat flux that would occur by pure conduction in case of concentric annulus, $\mathrm{Keq}=\mathrm{Nu} / \mathrm{Nu}_{\text {conc, }}$ (same as that used by Kuehn and Goldstein [2], Badr [3] and others). Shown also in the same figure are the experimental results of Kuehn and Goldstein [2] and numerical results of Chung et al. [19]. The figure shows very good agreement with both the experimental results and the numerical results for the distribution of equivalent conductivity along the inner wall. While the distribution along the outer wall shows very good agreement with the numerical results and satisfactory agreement with the experimental ones but with some observable deviation in the upper part of the annulus. Such a deviation maybe attributed in part to the less precision of measuring devices used in the experimental work and in part to the assumptions adopted in the numerical work. Same deviation has been also observed in the similar numerical works (see Prusa and Yao [5] and Chung et al. [19]).

The flow field and thermal fields are shown in Fig.8 for the cases of asymmetric annulus at $\lambda=0,30$, and 60 . The figure shows the existence of global flow circulation around the inner wall. The intensity of global flow can be inferred from the position of zero stream function line with respect to the inner wall. The closer the line to the inner wall, the less the intensity of global flow. Moreover, the figure shows that the global flow joins the left hand eddy in this case of negative eccentricities. The global flow, as it can be inferred is zero in case of $\lambda=90$ (as in Fig. 3a) and increases with the decrease of $\lambda$ to reach maximum in case of $\lambda=0$. The effect of increasing global flow intensity on the inclination of thermal plume above the inner wall can be also observed. The more the intensity of global flow the more the inclination of thermal plume in the direction of global flow circulation.

\section{CONCLUSIONS}

In this work the natural heat convection within an annulus formed between two eccentric long cylinders has been studied. The annulus inner wall is heated and maintained at constant temperature while the outer wall is cooled and maintained at constant temperature less than that of the inner wall. Beside Rayleigh and Prandtl numbers the heat convection process in the annulus depends on the inclination of ASL, radius ratio and eccentricity. The study considered a moderate range of Rayleigh numbers up to $10^{5}$ while Prandtl number is fixed at 0.7 . The angle of ASL is varied from zero (the horizontal position) to 90 (vertical position). The radius ratio is fixed at 2.6 while the eccentricity is varied between \pm 0.65 . The study has shown that as $\lambda$ increases $\overline{N u}$ increases for negative eccentricities while it deceases for positive eccentricities. The study predicted the global flow circulation within the annulus, which increases as annulus ASL moves from the vertical position to horizontal position. The study has also shown that the present numerical solution of 
the pure conduction problem is almost identical with the analytical solution which confirms the high accuracy of the numerical solution.

\section{REFERENCES}

[1] Kuehn T. H. and Goldstein, R. J., "An Experimental and Theoretical Study of Natural Convection in the Annulus between Horizontal Concentric Cylinders," J. Fluid Mech., 74, pp. 695-719, (1976).

[2] Kuehn T. H. and Goldstein, R. J., "An Experimental Study of Natural Convection Heat Transfer in Concentric and Eccentric Horizontal Cylindrical Annuli," ASME J. Heat Transfer, 100, pp. 635-640, (1978).

[3] Chakrabarti, S., Probert, S. D. and M. Shilton, J., "Optimal eccentric annuli (containing atmospheric-pressure air) for thermally insulating, horizontal, relatively cold pipes", Appl. Energy, vol. 14, pp. 257-293, (1983).

[4] Badr, H. M., "Study of laminar free convection between two eccentric horizontal tubes", Transactions of the Canadian Society for Mechanical Engineering, 7(4), 191-198, (1983).

[5] Prusa, J., and Yao, L. S., "Natural Convection Heat Transfer Between Eccentric Horizontal Cylinders," ASME, J. Heat Transfer 105, pp. 108-116, (1983).

[6] Guj, G. and Stella, F., "Natural Convection in Horizontal Eccentric Annuli: Numerical Study," Num. Heat Transfer 27, pp. 89-105, (1995).

[7] Guj G., lannetta S. and Moretti G., "Experimental analysis of thermal fields in horizontal eccentric cylindrical annuli”, Exp. Fluid, vol. 12, pp. 385-393, (1992).

[8] Mahfouz, F. M., 2009, "Buoyancy driven flow within an inclined elliptic enclosure", Int. J. of Thermal Sciences, 50, pp. 1887-1899, (2011).

[9] Wang, Y. Z. and Bau, H. H., "Low Rayleigh Number Convection in Horizontal, Eccentric Annuli," Phys. Fluids, 31(9), pp. 2467-2473, (1988).

[10] Cho, C. H., Chang, K.S., and Park, K.H.," Numerical Simulation of Natural Convection in Concentric and Eccentric Horizontal Annuli," ASME, J. Heat Transfer 104, pp. 624-630, (1982).

[11] Projahn, U., and Beer, H., "Prandtl Number Effects on Natural Convection Heat Transfer in Concentric and Eccentric in Horizontal Cylindrical Annuli," Heat and Mass Transfer, 19, 249-254, (1985).

[12] Adlam J. H., "Computation of two-dimensional time-dependent natural convection in a cavity where there internal bodies", Compt. Fluids, 14, pp. 141157, (1986).

[13] Stella, F. and Guj, G., "Vorticity-velocity formulation in the computation of fluids in multiconnected domains", Int. J. Numer. Methods Fluids, 9, pp. 1285-1298, (1989).

[14] Shu, C., Yao, Q., Yeo, K.S, "An efficient approach to simulate natural convection in arbitrarily eccentric annuli by vorticity-stream function formulation". Numer. Heat Transfer, A 38, 739-756, (2000).

[15] Shu, C., and Wu, Y.L., "Domain-Free Discretization Method for Doubly Connected Domain and its Application to Simulate Natural Nonvection in Eccentric Annuli," Comput. Methods Appl. Mech. Eng., 191, pp. 1827-1841, (2002) 
[16] Lee, T. S., Hu, G. S., and Shu, C., "Application of GDQ Method for the Study of Natural Convection in Horizontal Eccentric Anuuli," Num. Heat Transfer, Part A, 4, pp. 803-815. (2002).

[17] Badr, H. M., and Dennis, S. C. R., "Time-Dependent Viscous Flow Past an Impulsively Started Rotating and Translating Circular Cylinder," J. Fluid Mech. 158, pp. 447-488, (1985).

[18] Mahfouz, F. M. and Badr, H. M., "Flow Structure in the Wake of a Rotationally Oscillating Cylinder," ASME J. of Fluids Eng., 122, pp. 290-301, (2000).

[19] Chung, J. D., Kim, C.-J., Yoo, H., and Lee, J. S., "Numerical Investigation on the Bifurcative Natural Convection in a Horizontal Concentric Annulus," Num. Heat Transf., Part A, 36, pp. 291- 307, (1999).

\section{FIGURES}

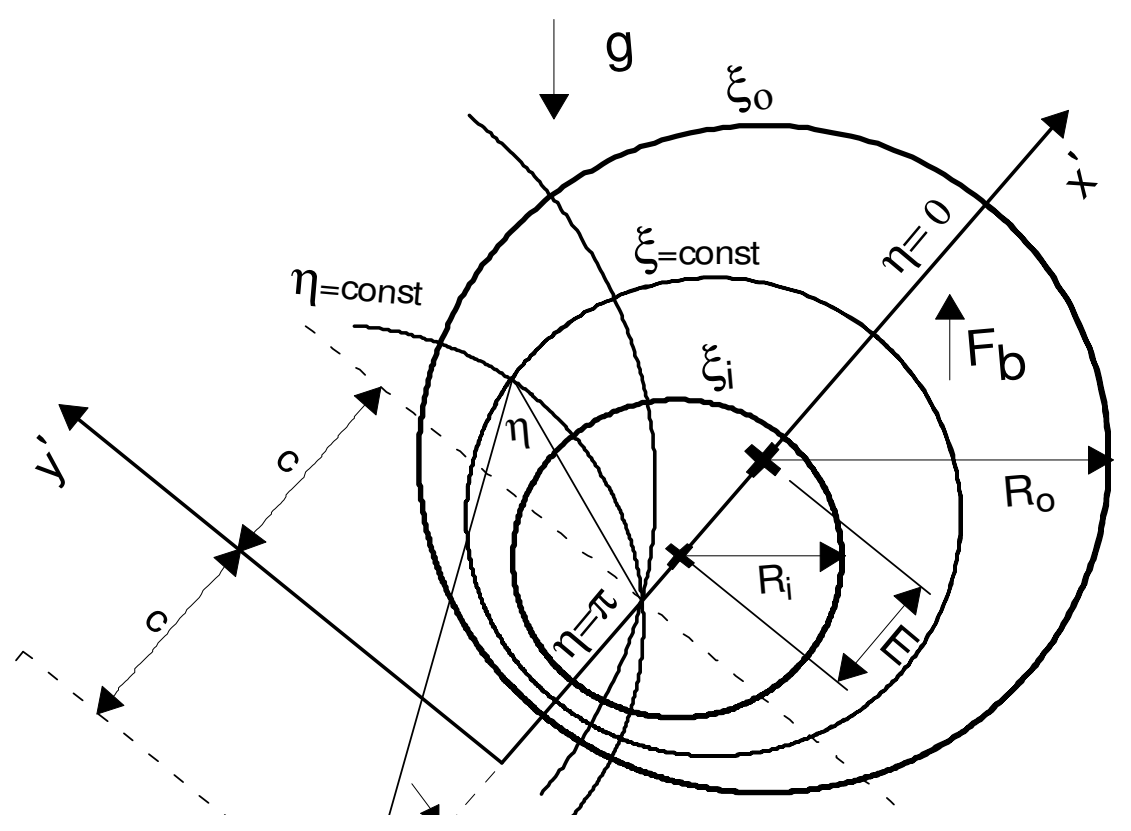

Fig. 1. Physical domain and coordinate system. 


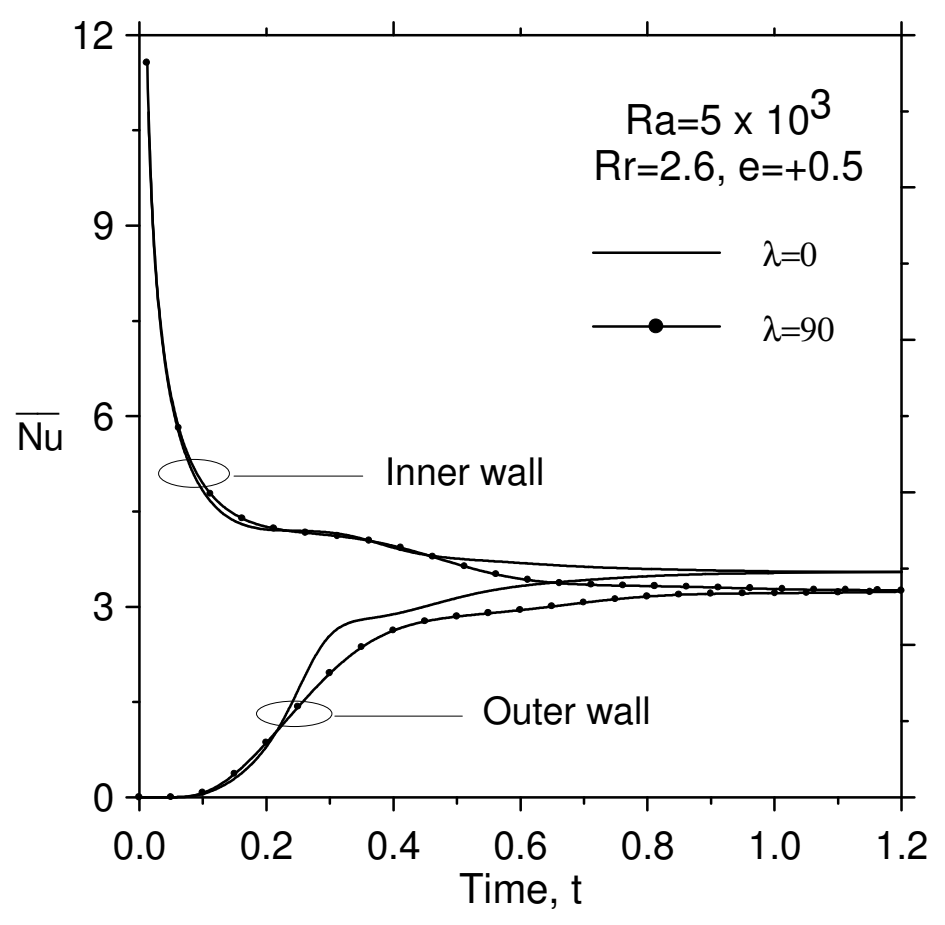

Fig. 2. The time development of $\overline{N u}$ at outer and inner walls of the annulus at $\mathrm{RaL}=5 \times 10^{3}, \mathrm{e}=+0.5$ and at $\mathrm{ASL}$ vertical $(\lambda=90)$ and $\mathrm{ASL}$ horizontal $(\lambda=0)$.

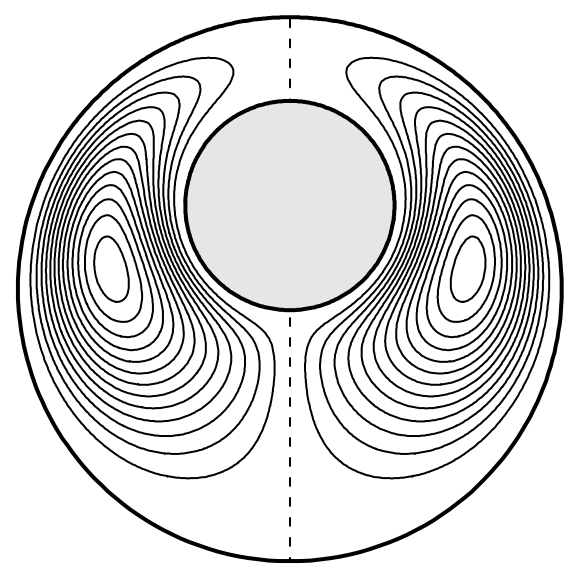

(a)

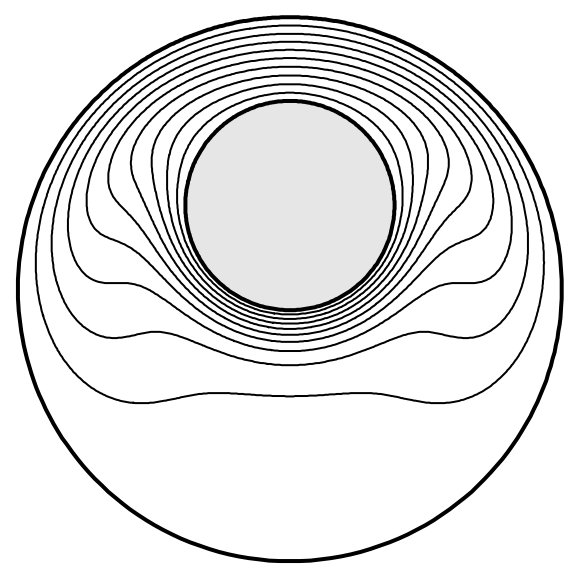

(b)

Fig.3. The Streamlines (left) and isotherms (right) for case of Ral $=5 \times 10^{3}, e=+0.5$ and

$$
\lambda=90.0
$$




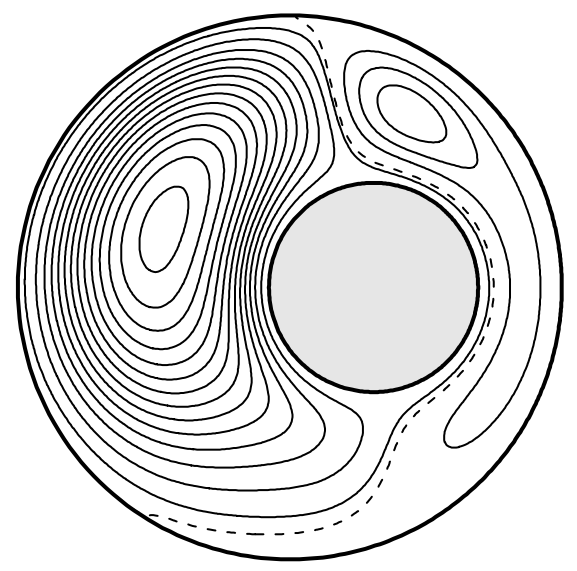

(a)

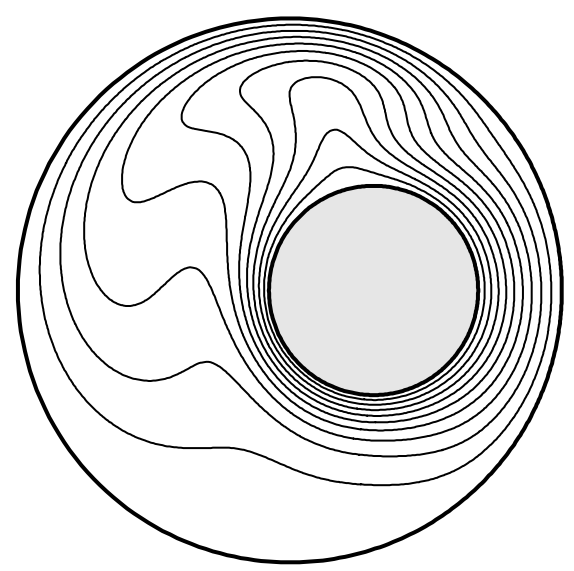

(b)

Fig.4. The Streamlines (left) and isotherms (right) for case of Ral $=5 \times 10^{3}, \mathrm{e}=+0.5$ and $\lambda=0.0$

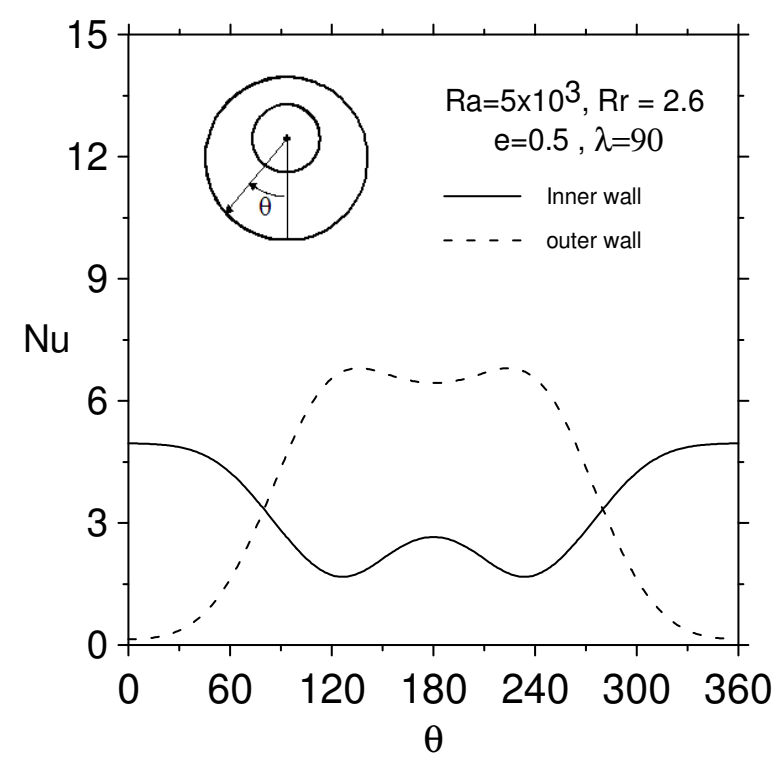

(a)

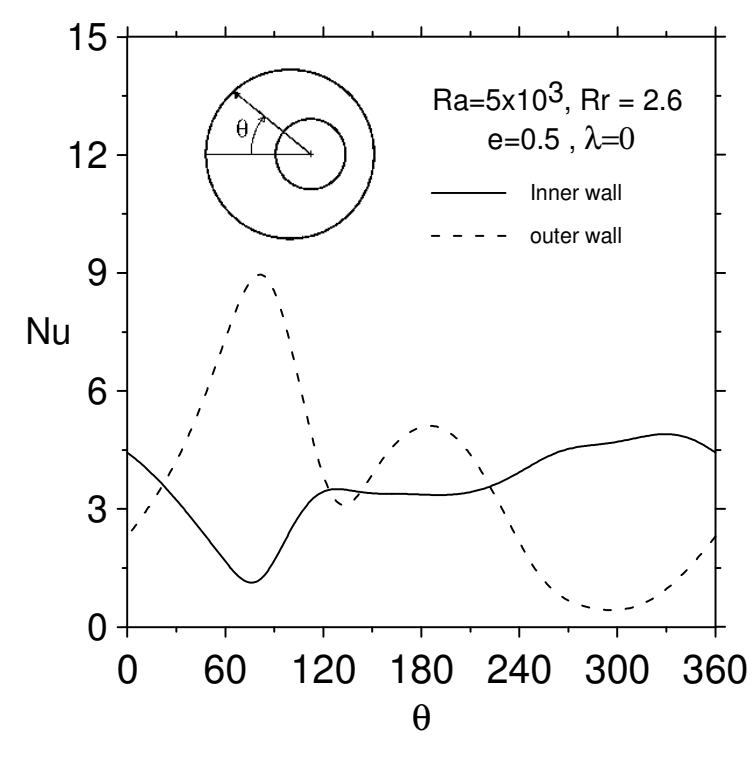

(b)

Fig. 5. Distribution of local Nusselt number along inner and outer walls at $\mathrm{RaL}=5 \times 10^{3}, \mathrm{e}=+0.5$ and a) $\lambda=90$ and b) $\lambda=0$. 


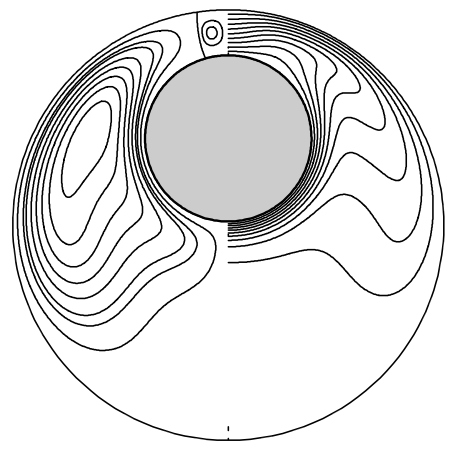

Num. isotherms (right) and streamlines (left)

Present

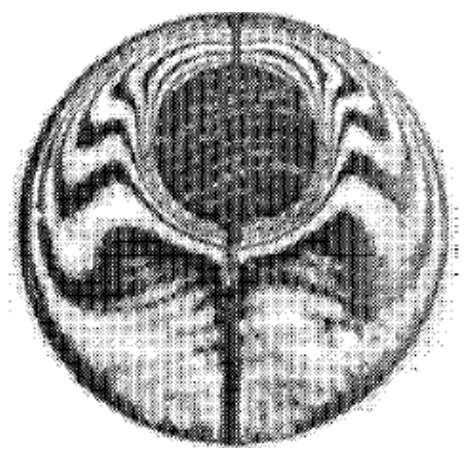

Exp. Isotherms

(right and left) of

Kuehn and Goldstein [2]

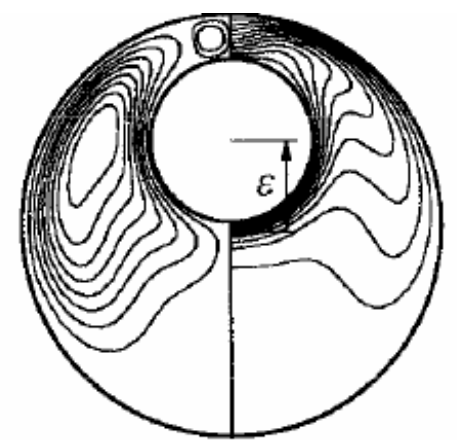

Num. isotherms(right) and streamlines (left) Chung, et al. [19]

Fig. 6. Comparison of present numerical results with previous experimental and numerical results at $\mathrm{RaL}=4.8 \times 10^{4}, \mathrm{Rr}=2.6, \lambda=90$ and $\mathrm{e}=+0.652$.

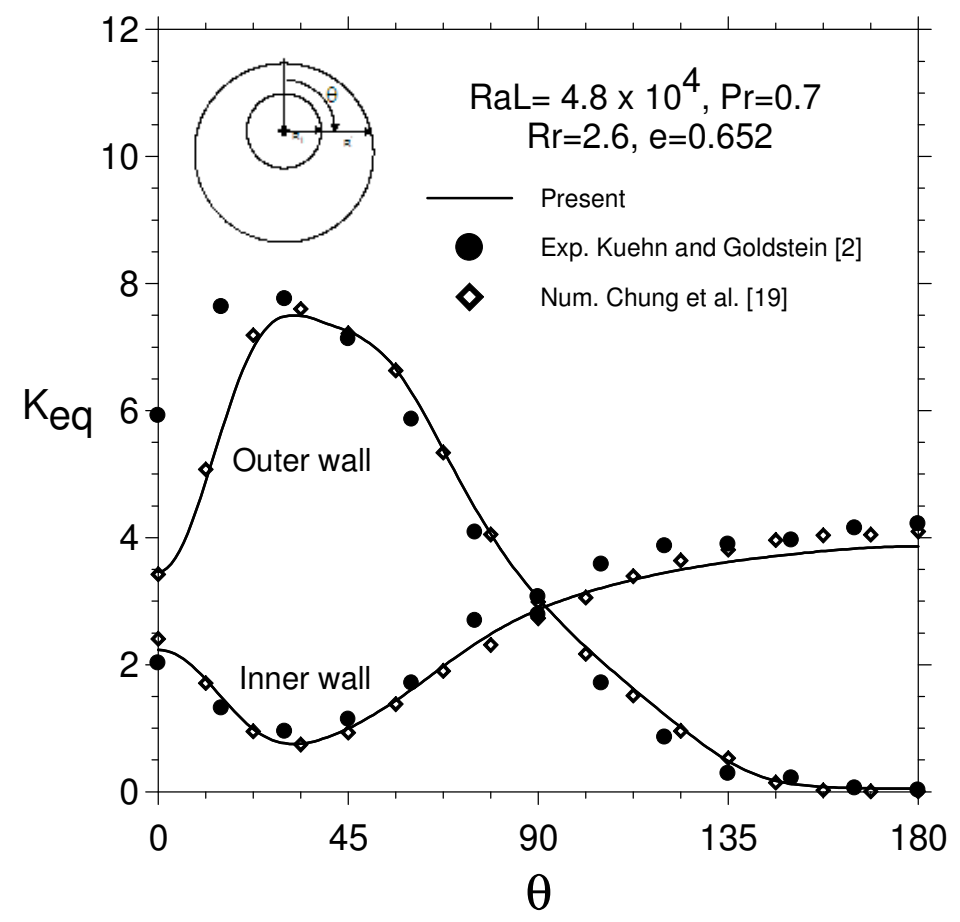

Fig. 7. Distribution of local equivalent conductivity along inner and outer walls at $\mathrm{RaL}=8 \times 10^{4}, \lambda=90$ and $\mathrm{e}=+0.652$. 
a) $\lambda=0$
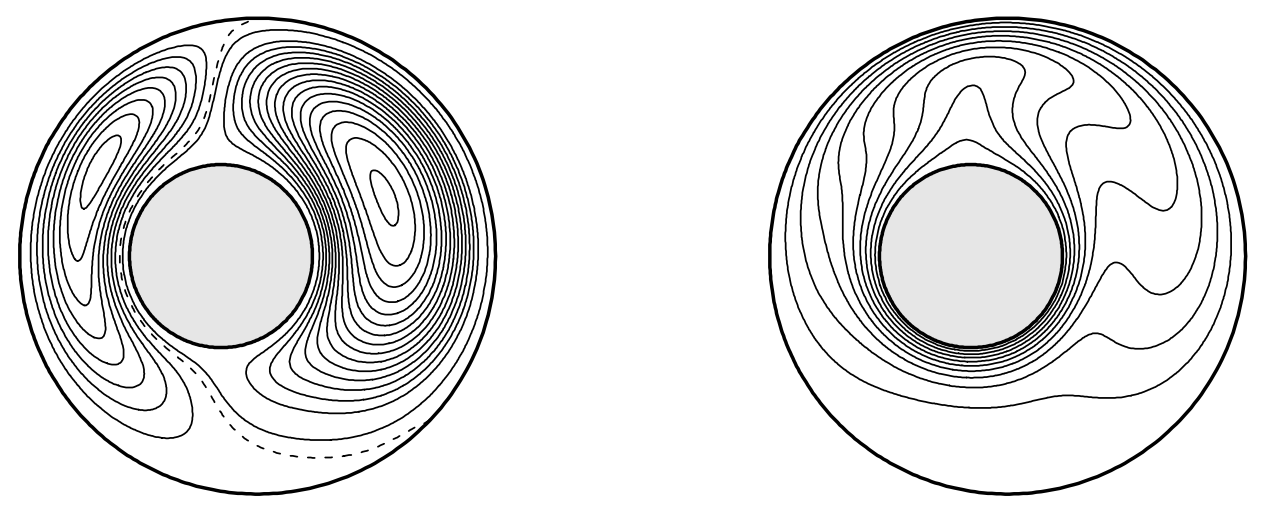

b) $\lambda=30$
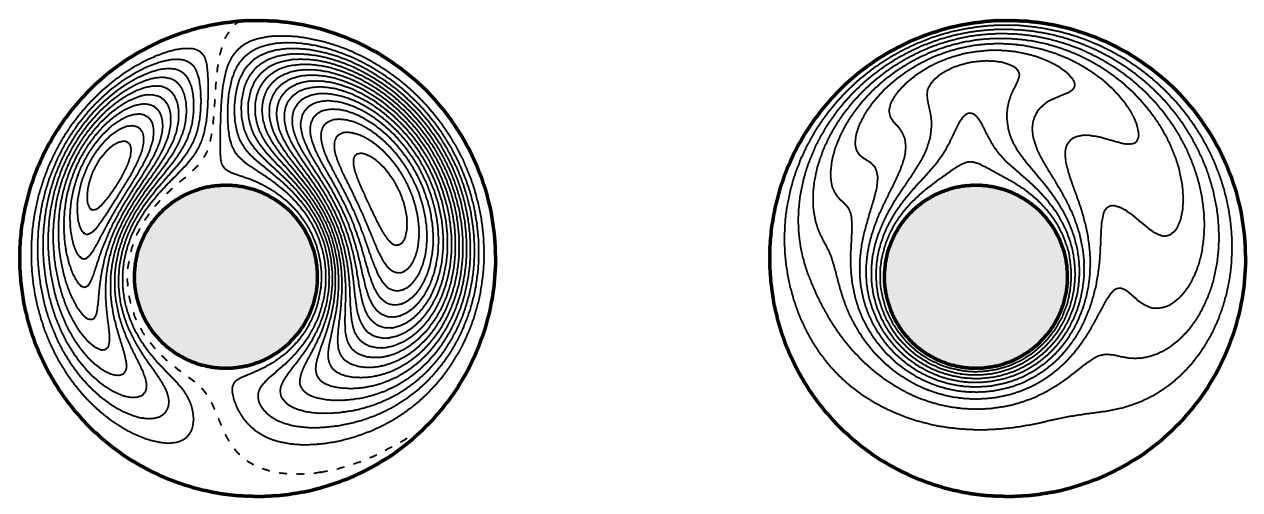

c) $\lambda=60$
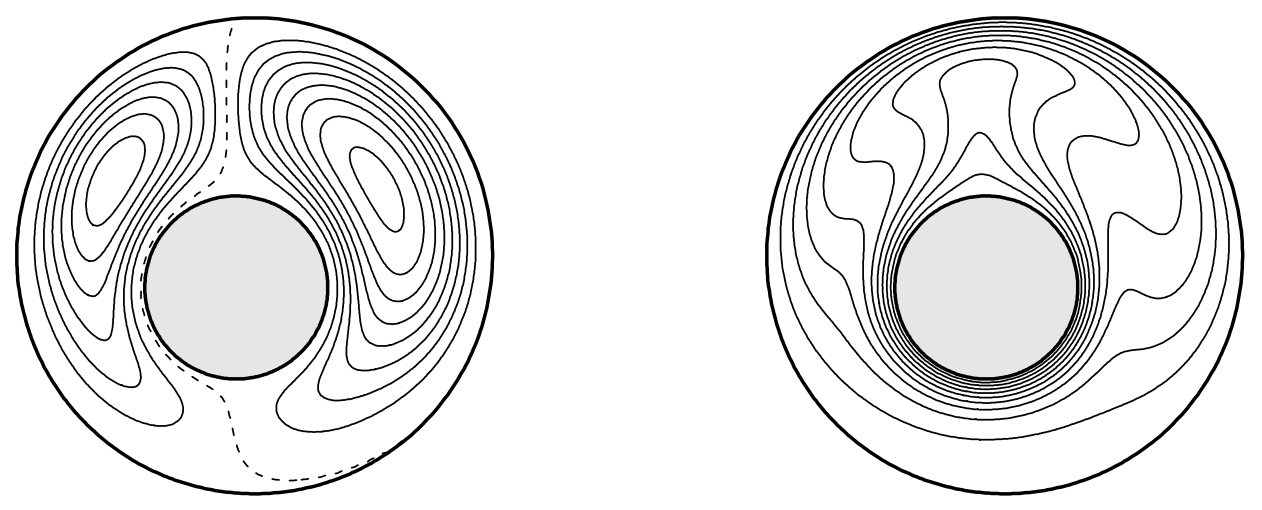

Fig. 8. The Streamlines (left) and isotherms (right) at $R a L=10^{4}, e=-0.25$ and at a) $\lambda=0 \quad$ b) $\lambda=30$ and c) $\lambda=60$ 